\title{
THE DISORGANIZATION OF BEHAVIOUR IN FATIGUE
}

BY

\author{
D. RUSSELL DAVIS
}

From the Medical Research Council Unit of Applied Psychology, Cambridge

(RECEIVED 6TH JANUARY, 1946)

MANY studies of mental fatigue have been governed by the expectation that its effects were to be found in a decline in the quantity or quality of the output, and tests have been employed in them involving the repetition of elementary operations. Bartlett (1943) contended, however, that effects related to real life were to be found in the disorganization of skilled activities, of which the work of every-day life largely consists. He therefore proposed that the organization of a relatively complex skill should be studied under conditions which might reasonably be assumed to be fatiguing. This was made possible by an ingenious apparatus, now called the Cambridge Cockpit, which was designed and built by the late Dr. K. J. W. Craik. Bartlett has described some of the results of the first experiments with this apparatus, which were carried out by Drew, and has shown how they throw light on the organizing functions of the central nervous system. He has referred mainly to the perceptual aspects of the disorganization. This paper, based upon further experiments by the author, amplifies Bartlett's account in several respects and deals mainly with the motor aspects.

\section{Method and Procedure}

The Cambridge Cockpit was like a Link trainer, used for the teaching and practice of instrument flying. It was operated, however, by a different system from that of the Link trainer and had several advantages over it for the present experimental purpose, particularly because the movements of the controls and the resulting movements of the instruments could be recorded graphically in a form which could be readily scored. The cockpit itself did not move, although the instruments responded realistically to the movements of the controls. Their effects were less complex than those of an aircraft, however, since only the primary effects were produced, but, in spite of this and other differences from an aircraft, the test was accepted as a reasonable one by all the experimental subjects, and the similarity to flying was an important factor in their motivation. On the other hand, elaborateness of the apparatus limited its applicability, and it was, in general, too specialized for any but aircrew subjects. The subjects of the experiments to be described were R.A.F. pilots, fit for flying duties at the time of the test. The subjects carried out an exercise in instrument flying, which was of the same form in all the experiments. A series of four manœuvres, together occupying ten minutes, was repeated between intervals of straight and level flying. Only the periods of the manœuvres were scored, and the scores of successive periods were compared with one another.

The records of the "side-slip" instrument proved to be of particular interest, and this instrument was recorded in all the experiments. The directional gyroscope, airspeed indicator and altimeter were recorded in some experiments. The instructions and the recording were so arranged that the perfect performance was recorded as a straight line, unaffected by errors of timing of the manœuvres. Three types of score were obtained from the records of the instruments and were measures of errors in the control of the machine. The number of deviations of an instrument reading, exceeding an arbitrary minimum divergence from the prescribed reading, was counted, and since the deviations had always to be terminated by action on the part of the subject, this number was called the "corrections" score. The total time for which an instrument showed a similar deviation was measured with a map-measurer and tamplet and was called the "total duration of errors." This score was divided by the "corrections" score to give as the quotient a third score, the "average duration of errors." Counters of a cyclometer type were attached to each of the controls and measured the total amount of movement. This score is referred to as the "movements" score. Graphic records were made of the elevator and aileron movements whenever it appeared useful to do so.

Various other observations, including the timing of manœuvres, were made systematically. In particular, a note was made if the subject forgot to reset the "fuel contents" indicator, an action which was necessary every ten minutes. The resetting of this indicator was an example of the several actions which had to be performed only occasionally and were liable to be forgotten. Various ratings were made systematically on the basis of impressions gained at the beginning, during the course or at the end of the test, and subjects were questioned carefully at the end of the test. The test was assumed to be fatiguing, the important factor in producing fatigue being regarded as the difficulty subjects encountered in attaining a standard of performance satisfying to themselves. They strove to attain a standard of accuracy which was at least partly derived from their flying experience, but because of the characteristics of the apparatus it was difficult to attain the standard required when flying an aircraft. In any event, subjects, whether pilots or not, were liable to fall short of the standard which they set themselves, however it may have been derived. Thus, their efforts to attain a satisfying standard were frustrated, and they suffered the experience of failure. In this situation they were obliged to perform a skilled task for a relatively long time.

\section{Results}

Drew (1941) tested a large group of pilots and found that "side-slip" scores increased considerably as the test continued. He worked mainly with a single score from each instrument, which was roughly equivalent to the product of the scores of "corrections" and "total duration of errors." The curve drawn from the means for successive ten minute periods of a two-hour test had a somewhat unexpected shape, since its gradient was at first positively accelerating and then became negatively accelerating. This result suggested that there were two phases of the effects of the test conditions. Moreover, at the part of the curve at which the gradient changed, the scatter of the scores increased considerably, and this fact suggested that there were 
considerable individual differences in the time of development of the second phase. Further evidence was sought on these points.

I. Thirty-four relatively inexperienced pilots carried out a two-hour test. Four periods of the test were compared with one another, each being of twenty minutes and comprising two series of manœuvres. Means were calculated from the sums of the scores of the individuals. In general, they had a rather high standard deviation, but the trends described below were significant on the statistical
Three other observations were considered to be of importance. It was seen from the graphic records that after the second period the aileron movements became few and large instead of many and small, as they had been, although the counter score remained the same. The errors in the instrument readings increased in size towards the end of the test, as Drew had also found, and there were few large errors in place of many small ones. Thirdly, the number of omissions in resetting the "fuel contents" indicator fell from the first to the third period, but

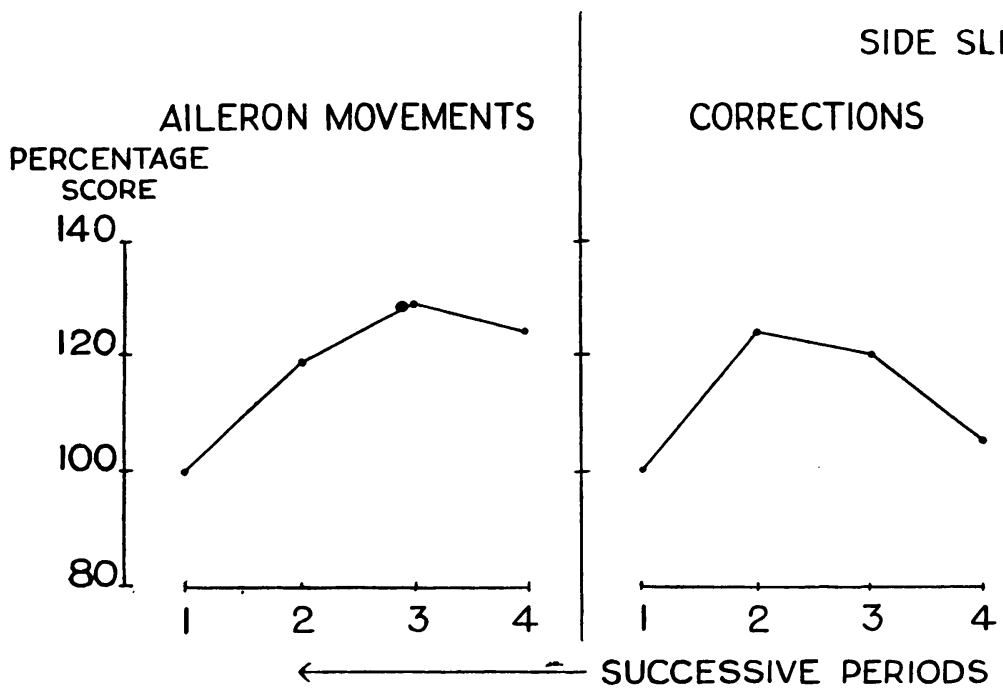

Fig. 1

Fig. 2 criterion that the "probability" was less than 0.05 .

It was found that the deterioration during the first part of the test affected control similarly in the three planes of movement, but towards the end of the test the " rolling plane" (side-slip) was the most affected. It was unlikely that this adverse effect on the "rolling plane" had any practical importance, but, as will be seen later, its selection was an artefact of the instructions. Aileron and side-slip scores were chosen for special study, and the means of these scores are displayed in Figs. 1-3.

The aileron "movements" score increased from the first to the second period, after which it did not alter significantly. The "corrections" score of the side-slip increased to a peak at the second period and fell to just above the initial level from the third to the fourth period. In contrast, the "average duration of errors" did not increase significantly above the initial level until it rose steeply from the third to the fourth period. The air-speed scores showed similar trends, but the trends were less marked and were not significant statistically. The "corrections" score of the directional gyroscope showed the same trend as that of the side-slip, although the peak at the third period was later. It fell significantly in the fourth period. The "average duration of errors" showed a different trend, however, since it decreased with each successive period. then increased significantly.

These results were considered to indicate two phases of the effects of the test conditions, which it is proposed to refer to provisionally as the phase of " overactivity" and the phase of " withdrawal." The first"phase of " overactivity", was transient and corresponded to the increases in the number of control movements and the number of corrections. The second phase, "withdrawal," corresponded to the decline in "corrections," to the increase of the "average duration " and size of errors and to the increase of omissions of the resetting of the "fuel contents" indicator. It appeared that in the first phase all aspects were affected, but the second phase affected only the "rolling plane" and the occasional actions.

Less systematic observations and the subjects' reports also pointed to two different phases. In the first part of the test, subjects felt tense, under strain or irritable and, sometimes, frankly anxious. Corrections were made with impatience and were hurried, and subjects felt that correction was urgent. They were often preoccupied with one or other aspect of the task which had acquired a temporary urgency. Their attention was "sticky" and was held by one aspect, instead of being distributed over the whole field of events. They were distractible in the sense that recent instructions or interventions gained the greater part of their attention. They tended to sit more erect and to grasp the controls more firmly. 
The task seemed more difficult, and this was sometimes felt to be unreasonable and was blamed on the working of the machine, while it was actually due to the change in the nature of the movements which the subjects made. Such sources of irritation and physical discomfort tended to gain their attention. Towards the end of the test they tended to relax, concentration failed and interest declined. The feeling of strain gave way to one of mild boredom, tedium or tiredness; sometimes, however, they felt satisfied with a standard of accuracy which was lower than when they were fresh. The task might appear easier because part of it was being neglected or, in other cases, because certain errors were accepted as inevitable faults of the machine and not as the responsibility of the subject. However, many retained a sense of dissatisfaction, but were no longer distressed by it and were more aware of tiredness and discomfort.

Some aspects of the task were better preserved than others, and this was shown in other experiments to depend upon the importance laid by the subject upon the several aspects. The more important an aspect was thought to be, the better it was preserved. On the other hand, control of the " sideslip" was particularly affected, because its importance was given less weight in the test instructions than other instruments. Side instruments, such as the "fuel contents "indicator, were neglected more than the central ones. Thoughts began to wander and careless mistakes were made. Manœuvres were omitted or carried out in the wrong order. Subjects might remark that their reactions had become slower, but it was possible that the delay occurred in the noticing, or appreciating, that an instrument needle had deviated, for the correction itself was often, although not always, brisk. Extraneous signals were variable in their effects.

The effects differed from one individual to another. In some cases there was a clear-cut transition from the first to the second phase. In others, there was a blend of the features of the two phases, a tendency to make larger and less appropriate control movements being reinforced by inattentiveness and, thence, by the larger size of errors before they were corrected. Indeed, quite a marked degree of inattentiveness might co-exist with excessive activity and irritability. Some subjects showed slight or no effects even at the end of the test. In some, the first phase was intense and prolonged, whereas in others it was slight and passed quickly. It was certain, however, that unless there was a change in the experimental conditions, an increase in the average duration and size of errors, an effect characteristic of the second phase was not followed by an increase in the activity.

II. The two phases were regarded as distinct reactions to the test conditions, the trends revealed by the group scores representing the two successive reactions of the average subject. Individual differences were relatively large and, since two reactions appeared to be involved, it was thought to be more satisfactory to separate individuals into two classes, according to which reaction was the predominant one, than to study the average trends of a heterogeneous group. Such a separation was made, therefore, a third class being added of those who showed little or no effects. This classification provided the opportunity of a closer linking of mental experience with behaviour.

In order to facilitate the classification of individuals, a test of 46 minutes was standardized, the whole session with instructions and practice occupying 70 minutes. For purposes of scoring, it comprised four similar periods of ten minutes. It was then applied to a large number of subjects, the main group comprising 355 fit pilots, all of whom had had more than 200 hours experience of flying. The mean scores were found to show the same trends as were shown in the first experiment, although the effects at the end of the test were less advanced by reason of the pilots' longer flying experience.

The subjects were classified on criteria which were based on their scores. About three-quarters (268), who showed slight or no changes, were put into the "normal" class. The remainder were then divided into "overactivity" (59) and "withdrawal " (28) classes on the criterion of the ratio of the " total duration of errors" to the " movements" scores of the fourth period. In the case of all the criteria the distribution of scores was a continuous one with a single mode, and the nearer one approached to the arbitrary line of division, the greater was the number of subjects. Nevertheless, the range of variation was considerable, as Fig. 4 and Table I show. By this method of classifying

TABLE I

\begin{tabular}{|c|c|c|c|c|}
\hline \multirow{2}{*}{ Class } & \multicolumn{4}{|c|}{ Test period } \\
\hline & First & Second & Third & Fourth \\
\hline 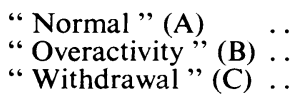 & $\begin{array}{l}16 \\
80 \\
60\end{array}$ & $\begin{array}{r}68 \\
295 \\
169\end{array}$ & $\begin{array}{r}42 \\
270 \\
52\end{array}$ & $\begin{array}{r}75 \\
509 \\
18\end{array}$ \\
\hline
\end{tabular}

"Movements" scores (elevator) of representatives of the three test classes.

individuals a more detailed picture was obtained of the characteristics of the two phases, or reactions of "overactivity" and "withdrawal."

The "Overactivity" Reaction.-The increase of activity was at first intermittent. It was the more frequent the greater the demands on the subject, as, for example, when he was required to carry out two or more manœuvres separated by only a short interval of "straight and level" flying. The tendency to excessive movement appeared to be related also to the irregular distribution of attention from one aspect of the task to another. The periods of excessive activity became gradually more frequent and lasted longer as the test progressed. Fig. 5 shows typical examples of the changes in the movements of the controls. At the beginning of the test, the movements were of small range and there was 

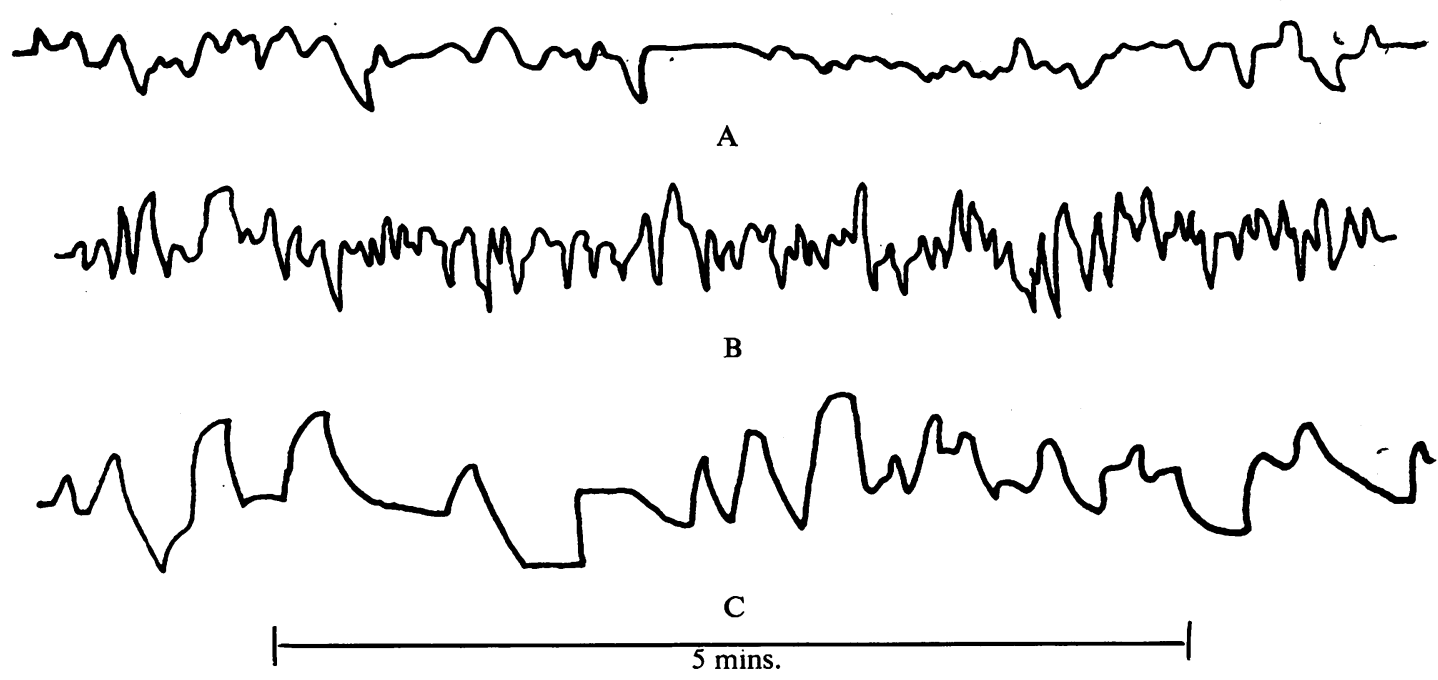

FIG. 4.-Extracts of the side-slip records of representatives of the three test classes.

A. " Normal " class.
B. " Overactivity" class.
C. "Withdrawal" class.

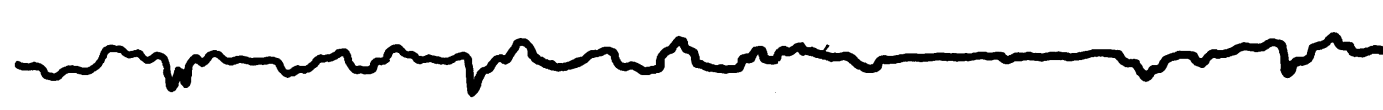

A
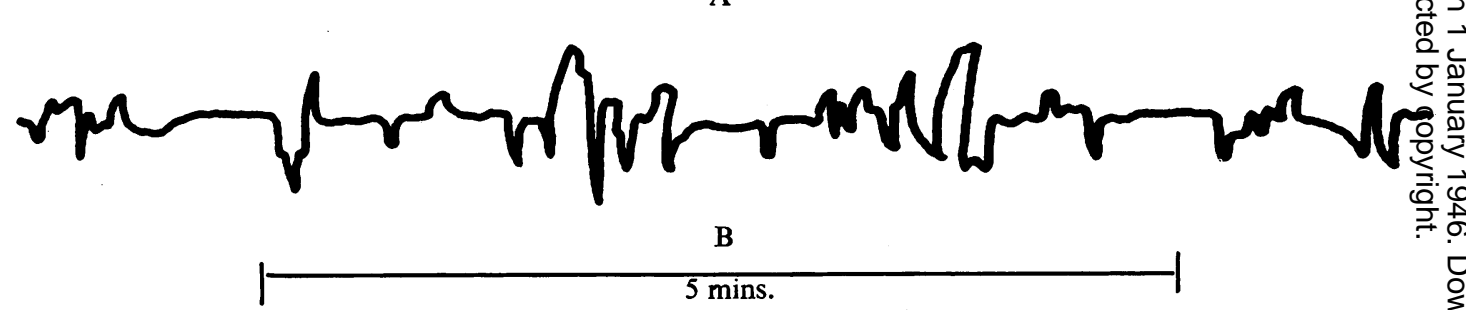

FIG. 5.-The " elevator" movements of a representative of the overactivity class.

A. At the beginning of the test.

B. 30 minutes after the start.

a ripple of small adjustments. At a later stage, the ripple had gone from the stretches of steady flying, and the record was interrupted by large, irregular movements. The principal changes appeared to be an increase in the extent and gradient of the individual responses to deviations of instrument readings, an increase in overcorrecting and, hence, in secondary responses, and an increase of restless movements of small range.

These other features were typically shown by subjects in the "over-activity" class: (i) Persistence. Their reports indicated that they continued to try to overcome the difficulties of the test. They were dissatisfied with their performance, but were not discouraged and were determined to improve it. (ii) Emotional disturbance. They experienced emotion, and signs of emotional disturbance, such as agitation and restlessness, were observed. They felt excited and, as has been stated, tense and under strain. Irritability was common. In some cases subjects reproached themselves, but in others irritability was directed at the machine or at the conditions of the test. It was often frankly aggressive, subjects

admitting the intention to injure the machine, but usually denying that the intention was expressed in $\overrightarrow{\hat{O}}$ action. Only a few subjects admitted to such expres- 3 sion, and, even in these cases, it has to be supposed that such actions were restrained to a varying degree ${ }^{-}$ or were insufficiently organized to be effective, sinces actual damage to the machine was rare from this cause. In other cases, however, random ando unorganized control movements reached such a . size and force that the mechanism was endangered.0 Violent manipulation of the fuel cock was the most common form of expression, and some subjects9 thumped violently on the instrument panel, com $\rightarrow$ plaining that an instrument was acting wrongly? Others swore vividly. (iii) Preoccupation with the test. Reports were obtained from many of these subjects that they had been preoccupied with the test after it was finished. Sometimes a subjecte returned later in the day to ask for a further oppor-o tunity of doing it or talked about it with surprising persistence. In several cases, when it was possible्⿱ to obtain reports, it was related that thoughts about? the test had recurred compulsively before going to sleep on the evening of the test. (iv) Flushing of 
the face. No special measures of recording bodily changes were employed, but coarse changes of various kinds were observed and rated. Thus, erectness of posture was observed and was typical of the "overactivity" class. There emerged one other striking difference between the classes. Flushing of the face was observed at the end of the test in 61 per cent. (36 subjects) of the " overactivity" class, whereas the proportion in the "normal" and "withdrawal" classes were 37 per cent. (99) and 11 per cent. (3) respectively. Visible sweating occurred in several of the "overactivity" class. Pallor was observed occasionally, but did not appear to be characteristic of either reaction. (v) Cumulative effects. Repeat tests were applied to some subjects at intervals of one or two days. The performance usually improved with each successive test, but, in some cases, where activity was excessive this class contained less than 10 per cent. of the whole group, the associated features were less definite and less constant than in the case of the "overactivity" class, and it is probable that the score changes were the common result of more than one psychological process.

In contrast to the restless striving of the " overactivity" class, the subjects of the "withdrawal" class gave the impression that they had lowered their standards of performance to a level well within their powers, and that there was no gap of relative failure between their attainment and aspiration. Errors, previously felt as threatening to their success, were not corrected and did not disturb them emotionally, and their judgment of the degree of accuracy to which they had attained was often faulty. They were not aware that they had left errors uncorrected and tended to be satisfied with their performance.

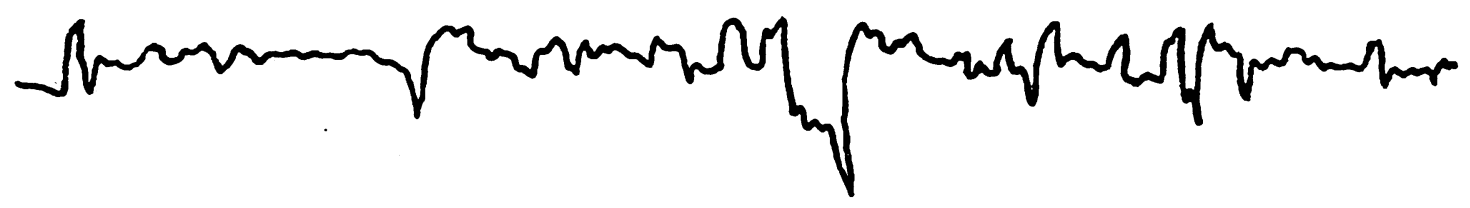

A

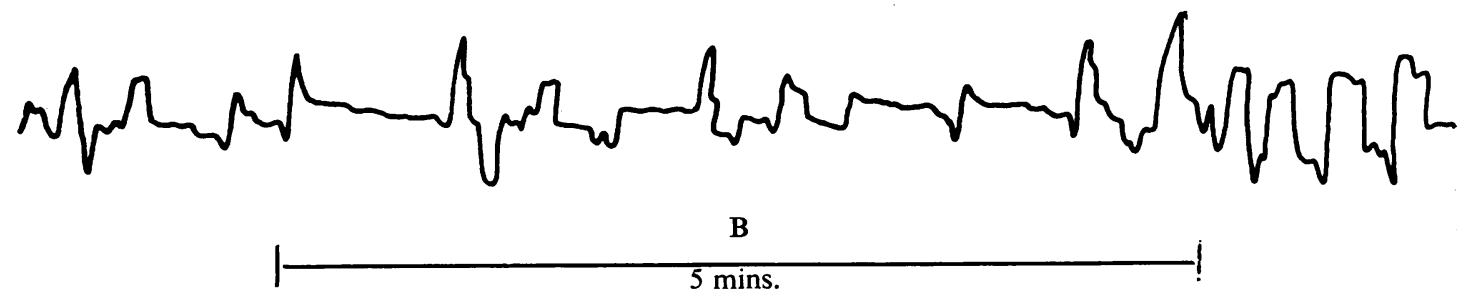

FIG. 6.-The " elevator" movements of a representative of the withdrawal class.

A. At the beginning of the test.

B. 30 minutes after the start.

in the first test, the increase of activity was more rapid in the later tests than during the first. This fact showed that the effects of the test conditions might be progressive from test to test, in the same way as they were progressive in the course of a single test.

The "Withdrawal" Reaction.-A definite increase in activity was observed in the majority of cases before the increase in the size and duration of the errors, characteristic of the " withdrawal " reaction, but the reaction developed early by comparison with the first experiment, because of the method of selecting the subjects for study.

The increase in the extent of the responses, characteristic of the " overactivity" reaction, persisted at first, while the instrument deviations became larger and lasted longer, but the responses were less hurried and less disturbed by restless movement, as Fig. 6 shows. While in some cases activity, as measured by the " movements" scores, declined to a very low level, generally the responses remained more extensive than at the beginning of the test, this change being related to the larger size of the deviations when they were corrected. Although
They admitted that they had become bored and had indulged in day-dreaming. It is probable that these effects were like those described in previous experiments on mental fatigue, in which a decline of output or loss of accuracy had been observed, complicated, in the way which Bartlett has discussed, by the greater complexity of the sensory pattern. As in these experiments, the effects were temporarily reversed by changes in the experimental conditions. Such changes revived the subjects' interest, although any improvement was short-lived.

Other subjects reported a loss of interest, but in a different setting. They were more aware of the deficiencies of their performance and were dissatisfied with it, but did not seem so distressed as those in the "overactivity" class. Several of th"m. were observed to be tense in posture and to be restless. Some blamed their inaccuracy on the apparatus or the conditions of the test. Blaming the apparatus or the test conditions was in the nature of an excuse and was not associated with the emotional disturbance characteristic of the " overactivity" reaction. The dissatisfaction of these subjects was not translated into effective activity; 
they were not provoked to irritability or anger and were apathetic. Their response to the demands of the test was an insufficient one, and they were discouraged.

\section{Discussion}

It was assumed $a$ priori that the test was fatiguing; that is to say, that the effects observed in the test were of the same kind as those occurring in states of fatigue, particularly in those which arise from the stress of flying. Although it was not the purpose of these experiments to prove this relationship, it may be remarked here that the assumption was compatible with the results obtained. Thus, many of the observed effects were similar to the symptoms reported by pilots in fatigue, e.g. " overcorrecting" and "stickiness" of attention, while flying, restlessness, irritability, preoccupation with flying and disturbance of sleep, on the one hand, and loss of concentration, loss of interest in and keenness for flying, apathy, discouragement, and depression, on the other hand. However, there is little direct evidence to show how skill in flying is affected in fatigue, except that accidents are said to be commoner, so that in this respect no comparison can be made.

The use of the term "fatigue" was provisional, except as a convenient rubric, since fatigue has no precise meaning in psychology and is applied to any of a series of states running all the way from true physiological exhaustion to psychological frustration uncomplicated by any detectable somatic disorder. When the experiments with the Cambridge Cockpit were planned, it was expected that disorganization of a skilled activity would be observed in a test involving prolonged effort at a difficult task. The disorganization having been demonstrated, the further definition of the conditions of its occurrence could be attempted and the term "fatigue," dispensed with. The experiments described in this paper prepared for this research by providing a more detailed description of the disorganization of the skilled activity than was available before, by showing that it was of two kinds, and by showing with what other mental and bodily effects each kind was associated.

The emotional disturbance, which was experienced by the subjects and of which the signs were observed, was considered to be the most important feature of the "overactivity" reaction. That emotion was provoked by the test conditions was not surprising, since subjects were obliged to carry out for a relatively long time a task in which they were unable to attain a satisfying level of performance, and the test, in this sense, constituted a " conflict" situation. It is probable that the restless and inappropriate movements, disrupting the skill of those in the " overactivity" class, were merely the reflection of the emotional disturbance and were essentially similar to the agitation and restlessness which are commonly observed in anxiety.

The effects comprising the " overactivity" reaction may be regarded as the results of a heightened expectancy, the relation of which to anxiety has been defined by Mowrer (1939). Expectancy, which $\underset{Z}{c}$ implies that learned behaviour has a striving or $\stackrel{\mathbb{Q}}{\mathcal{C}}$ goal-seeking character, describes the state of tension $\underline{\bar{O}}$ involving the anticipation of a reinforcement of a $\bar{Z}$ specific kind (Hilgard and Marquis, 1940). If $\stackrel{\mathbb{\Phi}}{\mathrm{C}}$ reinforcement does not follow, expectancy may be augmented at first (Mowrer, 1940). Thence, the magnitude of anticipatory responses is increased, 0 and anticipatory tension "spills over" into other motor channels and is displayed as restless activity. Geier and Tolman (1943), working with rats, have demonstrated a gradient of diffuse, random and restless activity, which increased as the goal was approached, but occurred only when the goalresponse was prevented.

The bodily changes, observed to be associated with the "overactivity" reaction, such as flushing $\overline{\frac{\bar{\rho}}{5}}$ of the face, have a counterpart in the decline of $\stackrel{\mathbb{Q}}{\varrho}$ palmar skin resistance, demonstrated in situations@ of " conflict" by Freeman and Pathman (1942). This effect was regarded as an indicator of altera-? tions in the " postural substrate of activity" and to $\vec{\omega}$ result from the vicarious discharge of " tension," $\stackrel{\circ}{\circ}$ when discharge in adaptive activity was prevented. Preoccupation with the task has been reported to occur in experiments involving conflict (Lewin and. Zeigarnik, quoted by Lewin, 1935; Rosenzweig, 1941). Aggressive activity was to be expected fropio the frustration-aggression hypothesis (Miller et apt, 1941).

The "withdrawal" reaction was so named $\Phi_{0}$ conform with the field theory of Lewin, since the effects were held to represent "withdrawal out the field of motivational conflict." Withdrawalifse said by Freeman (1939) to occur when tension risesfo to a level at which adjustment is no longer possible and to be characterized by behaviour of less thano appropriate motor requirement. This latter attribute was a feature of the reaction when it was fully developed, but, although some of the associated effects are not readily thus explained, the effects 0 reflected in the disorganization of the skill appeared $\overrightarrow{\vec{b}}$ to result from a reduction of expectancy. Expect 3 ancy may be reduced after reinforcement, when the goal-response is completed, but this did no? apply to the observed effects, since they followed upon the "overactivity" reaction, in which it was. supposed the goal of a satisfying standard of performance was not attained. It was more likely that: in this case, expectancy was reduced or inhibiteob by experimental extinction.

\section{Summary}

The skilled activity required by an exercise in instrument flying was observed to become dis organized in an experimental situation which was assumed to be fatiguing. The disorganization had two phases, in each of which it was associated witk characteristic changes of feeling. The two phases of the disorganization, with the associated effectso were considered to constitute two distinct reaction to the test conditions.

In the first phase, all parts of the work were 
similarly affected. Although there was little or no increase in their duration, errors became more frequent and were overcorrected. Activity became excessive; movements of the controls tended to be too extensive, and there was a large increase in restless and inappropriate movements. Attention was distributed irregularly over the work and was held by aspects which had acquired a temporary urgency.

Subjects felt under strain, dissatisfied and irritable, and they were preoccupied with the test after it was finished. Signs of emotion, such as agitation and restlessness, were observed, and there were indications that muscular tonus was increased. Flushing of the face was common, and visible sweating also occurred.

In the second phase, some parts of the work were affected more than others, those regarded by the subjects as of greater importance being the better preserved. Errors became less frequent, but their correction was tardy and they were larger. Movements of the controls were often as extensive as in the first phase, but were fewer. Concentration failed and interest flagged. Some subjects were satisfied with a lowered standard of accuracy, whereas others were dissatisfied with their attainment, but made an insufficient response to remedy it, either emotionally or in organized activity, and they were apathetic and discouraged. Postural changes were variable, but flushing, or sweating, was rare.

Acknowledgements.-The data on which this article is based have been obtained in the course of investigations on behalf of the Flying Personnel Research Committee of the R.A.F., and it is a pleasure to acknowledge the facilities afforded by Sir Harold Whittingham, K.C.B., K.B.E., Director-General of the Medical Service of the R.A.F. My thanks are also due to the many members of the R.A.F. and W.A.A.F. to whom I am indebted for invaluable assistance at all stages of the work. I was personally supported by the Medical Research Council.

\section{REFERENCES}

Bartlett, F. C. (1943). Proc. roy. Soc., B 131, 247.

Drew, G. C. (1941). Unpublished report.

Freeman, G. L. (1939). Psychol. Rev., 46, 226.

Freeman, G. L. \& Pathman, J. H. (1942). J. exp. Psychol., 30, 161.

Geier, F. M. \& Tolman, E. C. (1943). J. comp. Psychol., 35, 197.

Hilgard, E. R. \& Marquis, D. G. (1940). Conditioning and Learning, N. York : Appleton Century Co.

Lewin, K. (1935). A Dynamic Theory of Personality. London: McGraw Hill Publ. Co.

Miller, N. E. et al (1941). Psychol. Rev., 48, 337.

Mowrer, O. H. (1939). Ibid., 46, 553. (1940). Psychol. Monogr., 52, No. 233. Princeton N.J. Psychological Review Co.

Rosenzweig, S. (1941). Psychol. Rev., 48, 347. 